\title{
L'agriculture écologiquement intensive. Une approche économique
}

Ecologically intensive agriculture: An economic approach

\section{Mohamed Ghali, Karine Daniel, François Colson et Stéphane Sorin}

\section{(2) OpenEdition}

\section{Journals}

\section{Édition électronique}

URL : http://journals.openedition.org/economierurale/4338

DOI : $10.4000 /$ economierurale.4338

ISSN : 2105-2581

\section{Éditeur}

Société Française d'Économie Rurale (SFER)

\section{Édition imprimée}

Date de publication : 15 mai 2014

Pagination : 83-99

ISSN : 0013-0559

\section{Référence électronique}

Mohamed Ghali, Karine Daniel, François Colson et Stéphane Sorin, «L'agriculture écologiquement intensive. Une approche économique », Économie rurale [En ligne], 341 | mai-juin 2014, mis en ligne le 15 mai 2016, consulté le 30 avril 2019. URL : http://journals.openedition.org/economierurale/4338 ; DOI : 10.4000/economierurale.4338 


\title{
L'agriculture écologiquement intensive Une approche économique
}

\author{
Mohamed GHALI •École supérieure d'Agriculture d'Angers, LARESS; Université d'Angers, GRANEM \\ m.ghali@groupe-esa.com \\ Karine DANIEL • École supérieure d'Agriculture d'Angers, LARESS ; INRA Smart-Lereco UR1134, Nantes \\ François COLSON • Agrocampus-Ouest, Rennes \\ Stéphane SORIN • Terrena, Ancenis
}

L'agriculture française doit relever un double défi, environnemental et productif. Suite au Grenelle de l'environnement, le terme d'Agriculture Ecologiquement Intensive (AEI) est apparu comme un concept susceptible de répondre à ce double défi. Cet article analyse le concept d'AEI, son émergence et ses spécificités, en privilégiant une approche économique. II discute la pertinence des notions d'intensification écologique et de productivité des ressources naturelles à l'échelle de l'exploitation. II présente l'analyse de l'efficience productive des exploitations comme un cadre méthodologique pour l'évaluation de la démarche AEI.

MOTS-CLÉS : agriculture écologiquement intensive, intensification écologique, ressources naturelles, efficience productive, efficience environnementale.

\section{Ecologically intensive agriculture: An economic approach}

French agriculture is now facing a dual challenge of environmental sustainability and productivity. Further to the Grenelle Environment Conference (Environment roundtables organized in 2007 by the French government), Ecologically Intensive Agriculture (EIA) has emerged as a new concept to reach this dual challenge. This paper uses an economic approach to analyse the emerging concept of EIA and its specificities. It discusses the relevance of ecological intensification and natural resources productivity concepts at the farm level. It shows the measurement of productive efficiency of farms as a methodological framework for assessing the EIA approach. (Q16, Q29, Q32, Q40, Q57)

KEYWORDS: ecologically intensive agriculture, ecological intensification, natural resources, productive efficiency, environmental efficiency.

L 'intensification de l'agriculture au $\mathrm{XX}^{\mathrm{e}}$ siècle a permis aux pays développés de satisfaire leurs besoins alimentaires par une simplification des systèmes et une homogénéisation des pratiques. Ce modèle de développement a largement contribué à l'augmentation des volumes de production par unité de surface et d'emploi, et dans une moindre mesure par unité de capital et de consommation intermédiaire. Cette croissance a été jusque récemment évaluée par rapport aux seuls facteurs, terre, capital, travail et consommations intermédiaires, ignorant l'utilisation des ressources naturelles et leur pérennité. Ceci est dû principalement à la méconnaissance des limites de leur exploitation aux XIX ${ }^{\mathrm{e}}$ et début du $\mathrm{XX}^{\mathrm{e}}$ siècle.

Malgré ses succès, l'agriculture a été progressivement confrontée à des problèmes écologiques, économiques, sociaux et éthiques (Kiley-Worthington, 1981). Elle doit aujourd'hui relever des défis pouvant apparaître opposés ; protéger l'environnement et produire plus pour satisfaire l'accroissement de la demande. Ainsi, l'agriculture doit opérer une mutation, basée sur des nouvelles technologies et pratiques fondées sur une agronomie et une écologie scientifique où les agriculteurs doivent gérer la production et les écosystèmes (Pretty, 2008). 
Ainsi, ce concept de " révolution doublement verte » a été reformulé en France sous le terme d'" Agriculture écologiquement intensive » (AEI) à la suite du Grenelle de l'environnement (Griffon, 2007). Il est repris par les dirigeants d'une coopérative de l'Ouest de la France pour proposer à ses adhérents et à ses salariés une stratégie de développement qui répond aux enjeux environnementaux, en maintenant les volumes de production garants de l'équilibre économique des exploitations et des filières. L'AEI connaît un réel intérêt de la part des industriels, des agriculteurs et des organisations professionnelles notamment dans l'Ouest de la France, mais elle suscite aussi certaines critiques (Bonny, 2011 ; Goulet, 2012).

Jusqu'alors, le concept d'AEI a été illustré par des expériences de terrain, des pratiques agronomiques et doit faire l'objet d'un travail de conceptualisation scientifique, notamment économique afin de monter en généralités, tout en tenant compte de la diversité des milieux dans lesquels opèrent les exploitations agricoles (Bonny, 2011).

Dans cet article, nous proposons une lecture approfondie du concept d'AEI. Nous exposons dans la première partie l'intérêt porté à celui-ci, ses principes de base et leurs rapports avec la notion de la durabilité agricole. Dans la seconde partie, nous apportons à travers un cadrage théorique, des éléments de discussion et de compréhension de l'intensification écologique en économie agricole.

\section{L'agriculture écologiquement intensive Un concept original}

\section{Intérêt et principes de I'AEI}

L'intérêt pour l'AEI s'explique par deux raisons principales, (i) il n'oppose pas la préservation des ressources environnementales et le maintien ou l'amélioration des rendements, (ii) il ne se réfère pas à une liste de techniques agricoles ou à un type d'exploitation de référence, mais à une démarche d'évolution des pratiques mises en œuvre par les agriculteurs engagés dans des processus d'innovation. L'AEI se présente comme une démarche en se basant sur l'utilisation des capacités spécifiques des écosystèmes (Griffon, 2007, 2013). Ceci constitue une originalité qui ne se résume pas à un système de techniques spécifiques, mais qui peut englober un ensemble de techniques provenant de différents modes de production et implique l'ensemble des métiers agricoles. La mise en œuvre de l'AEI place les agriculteurs au cœur du processus d'expérimentation. À cet effet, une coopérative de l'Ouest de la France a dénommé ses adhérents les plus innovants «sentinelles de la terre ». Ces derniers forment un réseau d'exploitants et testent de nouvelles solutions techniques, y compris des combinaisons complexes (changements d'itinéraires et de systèmes techniques). L'expérimentation qu'ils mettent en œuvre permet une adaptation à la diversité des conditions pédoclimatiques et une meilleure diffusion des innovations sur le territoire de la coopérative. Cette initiative constitue une rupture par rapport aux discours et pratiques dominants et présente la relation à l'environnement et aux équilibres naturels comme un atout à mieux valoriser et non plus comme un obstacle au développement et la compétitivité des activités agricoles. De plus, le circuit de l'innovation et de la diffusion du progrès technique n'est plus conçu comme descendant des laboratoires de recherche et des firmes d'approvisionnement vers les exploitants agricoles, mais comme un processus itératif prenant en compte les initiatives des agriculteurs pour expérimenter la diversité des territoires, leur potentiel pédoclimatique et écologique. De ce fait, le mouvement de cette coopérative constitue un trait d'union entre la théorie de l'intensification écologique et la mise en relation avec la réalité du terrain. 
RECHERCHES

L'intérêt pour ce concept est donc réel, cependant, ni ses bases scientifiques ni ses performances techniques et économiques ne sont bien connues. Ainsi, nous analysons le concept d'AEI, son fondement théorique, les notions qu'il mobilise et discutons l'intérêt économique éventuel qu'il peut engendrer.

\section{Un concept mobilisant agronomie, écologie et économie}

Selon Griffon (2007), le principe de base de l'AEI est l'intensification écologique qui est l'obtention d'un rendement plus élevé par unité de biosphère pour un ensemble d'objectifs de viabilité recherché. Ainsi, l'AEI peut être appréhendée comme un système de production caractérisé par un ensemble de techniques et de technologies ayant pour objectif d'intensifier les fonctions d'un écosystème de production, permettant de maximiser la production agricole et les aménités environnementales, de réduire les externalités négatives et d'améliorer la gestion des ressources naturelles.

L'AEI repose sur l'idée que les mécanismes biologiques naturels, au cœur des processus productifs, peuvent être mieux valorisés (figure 1). Elle suppose une gestion des techniques agricoles et des aménagements du paysage plus complexe qu'en agriculture conventionnelle, même si elle reste dans une logique de maintien

Figure 1. Comparatif de l'agriculture conventionnelle et de l'agriculture dans une démarche AEI

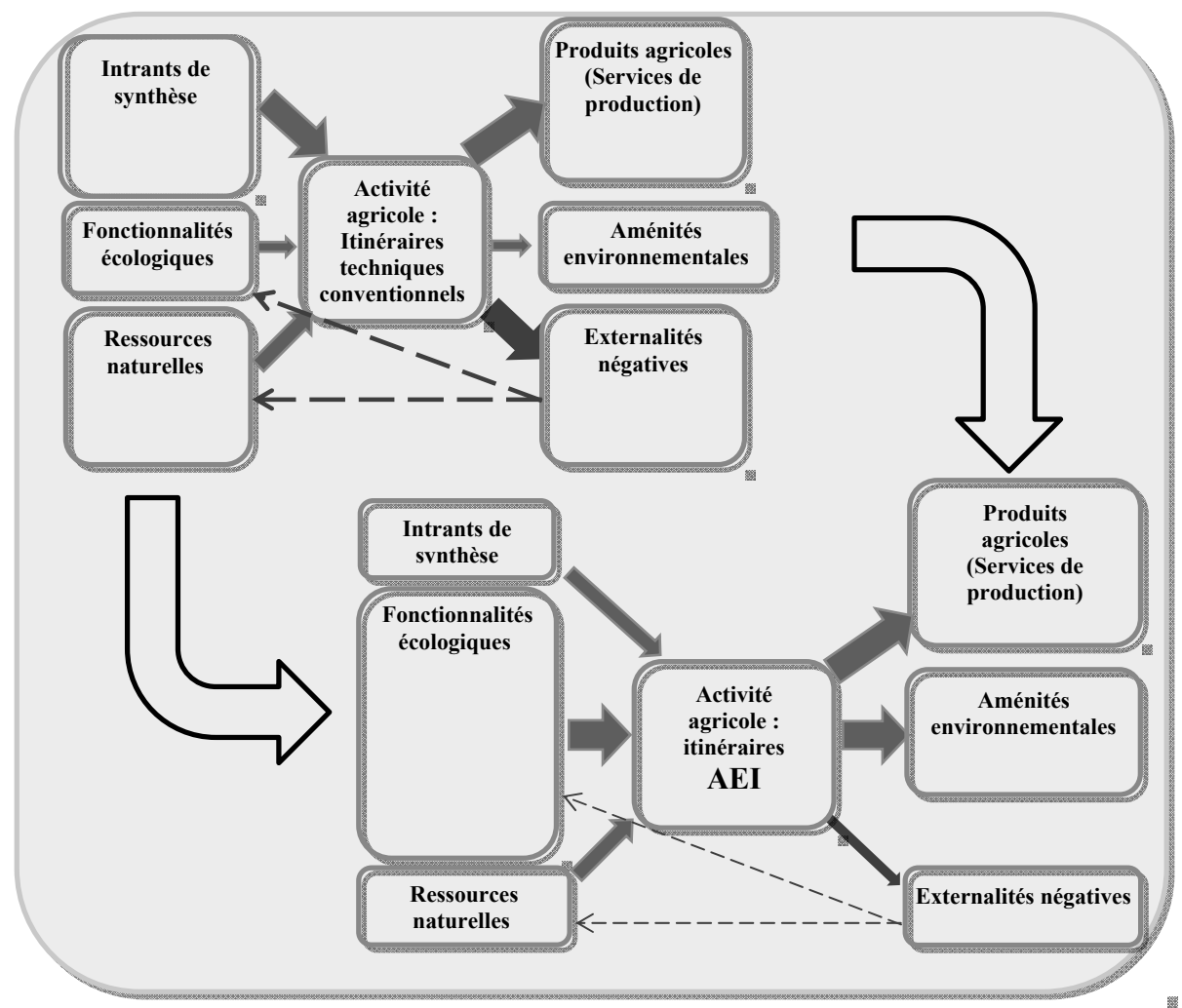

Source : adaptée et modifiée à partir de Griffon (2013). 
ou d'augmentation des rendements et des revenus. Il s'agit de trouver des innovations systémiques qui sont attachées à chaque écosystème tel qu'il est dans la réalité, mais qui sont également délicates à concevoir (des itinéraires techniques à bas intrants) que des innovations ponctuelles (une nouvelle variété végétale par exemple). Ces innovations doivent permettre aux agriculteurs de se déplacer d'un état stable antérieur " agriculture conventionnelle » à un état stable futur « agriculture durable» (Griffon, 2012). Ainsi, l'AEI s'inscrit dans une dynamique de changement nécessitant un environnement propice. En effet, les pouvoirs publics peuvent contribuer au développement de l'AEI en investissant dans la recherche et le développement et en participant à la réduction de l'incertitude et des risques pris par les agriculteurs, donc en stabilisant leur environnement économique.

L'enjeu de l'AEI est donc que les agriculteurs se réapproprient l'optimisation des fonctionnalités des écosystèmes, en réduisant le recours aux intrants de synthèse et aux ressources non renouvelables, sans pénaliser la production et la viabilité des exploitations. Ainsi, la réussite de la démarche AEI peut améliorer l'efficience productive des exploitations et générer une rente indirecte de l'innovation qui peut engendrer des produits de meilleure qualité environnementale sans augmentation des prix. Ceci stimulera une nouvelle demande pour des produits innovants, qui augmentera le volume des ventes et assurera une meilleure formation de la rentabilité économique.

Ces éléments structurant l'AEI ont été abordés dans des travaux portant sur les formes d'agriculture durable ou sur l'intensification écologique. Pretty (2008) insiste sur l'importance de trouver de nouvelles technologies et pratiques pour assurer une agriculture durable et qui doivent être adaptées aux conditions locales de l'exploitation. Il propose des principes fondamentaux de la durabilité qui rejoignent l'AEI sur de nombreux points tels que la nécessité d'intégrer les processus biologique et écologique dans la production agricole, celle de réduire les intrants non renouvelables, la promotion et la valorisation des compétences des agriculteurs. Le rapport du Millenium Ecosystem Assessment (2005), Balmford et al. (2008), Lavorel et Sarthou (2008), démontrent l'importance des services écosystémiques et de la biodiversité pour le bien-être humain. Gliessman (2004) évoque l'intégration des processus agro-écologiques dans les systèmes de cultures, Cassman (1999, 2008), la FAO (2009), cités dans Bonny (2011), font référence, sous différentes formes, à l'intensification écologique pour améliorer la production agricole. En ce qui concerne le risque et l'incertitude liés à l'innovation, Ridier et al. (2013) montrent que la variabilité des rendements et le facteur temps de travail peuvent être des barrières à l'adoption des pratiques agrienvironnementales, de même les travaux de Chambers et al. (1989) ; Uphoff (1998), Olsson et Folke (2001) montrent que les systèmes agricoles avec des niveaux élevés en capital social et humain sont plus en mesure d'innover dans un contexte d'incertitude.

L'AEI correspond aujourd'hui à un ensemble d'expériences ponctuelles qui doivent être mises en commun et validées pour prouver sa capacité à assurer la viabilité des systèmes agricoles. Elle souffre d'un manque d'ancrage, en particulier sur le plan économique, pour lui donner une base crédible. Les seuls éléments économiques auxquels font référence l'ensemble des systèmes agricoles alternatifs est la théorie mathématique de viabilité permettant d'intégrer les caractéristiques du développement durable, notamment celle de l'équité intergénérationnelle prenant en compte un horizon temps infini (Aubin, 1991). Cette théorie ne s'attache 
pas à l'utilisation optimale des ressources, mais considère l'ensemble des utilisations viables de la ressource, la viabilité étant définie par le respect d'objectifs économiques, écologiques et sociaux (Martinet, 2010 ; Martinet et Doyen 2007). Griffon (2007) se réfère à cette théorie pour définir les limites de l'intensification écologique. Il parle de viabilité de l'écosystème quand on peut, par sa gestion, garantir le renouvellement des ressources naturelles renouvelables (eau, biodiversité, structure et fertilité du sol) et équilibrer les recettes et les dépenses. Ce cadre théorique de la viabilité est important dans la mesure où il permet d'identifier les champs des possibilités d'évolution des exploitations agricoles engagées dans l'AEI. Ces dernières doivent pouvoir évoluer vers et dans ce cadre de " contrôle viable » (Martinet, 2010) en se basant sur l'adoption des technologies et des pratiques agricoles issues de l'intensification des processus biologiques et écologiques.

Cependant, les instruments d'analyse et d'évaluation de la démarche restent à identifier et plusieurs questions peuvent alors se poser, à savoir : comment peut-on apprécier l'efficacité économique et environnementale des changements de technologies et de pratiques agricoles préconisés par l'AEI ? Et comment peut-on déterminer l'efficience des exploitations agricoles en termes d'utilisation des fonctionnalités écologiques et des ressources naturelles ?

\section{Analyse économique de l'AEI}

\section{Intégrer l'intensification écologique et les ressources naturelles}

L'AEI interpelle par la juxtaposition des termes « écologie » et « intensif». Le terme intensif emprunté à l'économie veut que l'agriculture de demain soit intensive en écologie. Or la définition sur laquelle il y a le plus de consensus est celle de Tirel $(1983,1987)$ qui rappelle qu'en microéconomie l'intensification se réfère à une unité de facteur de production à laquelle on combine des quantités accrues d'autres facteurs. Il distingue, par rapport au facteur limitant «terre ${ }^{1}$, les formes d'intensification appelées, par les AngloSaxons, Labor-intensive, dans lesquelles l'exploitation intensive du sol est obtenue par un accroissement de la main-d'œuvre, et les formes Capital-intensive dans lesquelles l'intensification est obtenue par une augmentation du capital (consommations intermédiaires, matériels, bâtiments) (Tirel, 1983 ; Tirel, 1987). Ainsi, une agriculture écologiquement intensive repose sur une forme d'intensification basée sur une utilisation accrue des fonctionnalités écologiques par rapport aux facteurs limitants que sont les ressources naturelles, dont la terre. Ainsi, l'originalité du concept AEI est de spécifier les fonctionnalités écologiques et les ressources naturelles (RN) comme des facteurs de production spécifiques et qu'au-delà des critères habituels, la performance économique doit être évaluée par le niveau de productivité de ces facteurs.

Analyser cette hypothèse nécessite en amont d'éclaircir les relations entre les fonctionnalités de l'écosystème et les ressources naturelles afin d'évaluer la possibilité de les considérer comme des facteurs de production.

\section{Les fonctionnalités écologiques de l'écosystème}

Elles se définissent comme les processus biologiques de fonctionnement et de maintien de l'écosystème et assurent sa capacité à faire face à des perturbations et à se maintenir dans un état favorable à la production (MEEDDM, 2010). Selon Balmford et al. (2008), ces fonctionnalités correspondent à des processus participant à la production

1. Butault et al. (1985) considèrent l'intensification comme « la croissance du produit par unité de surface ». 
de services écologiques ayant un impact direct sur le bien-être humain. Ils distinguent deux types de processus : (i) les processus de base des écosystèmes, Core' ecosystem process (ex. cycle des nutriments (azote, phosphore, carbone), cycle de l'eau, interaction inter- et intra-organismes, la production de biomasse, etc.) et (ii) les processus bénéfiques de l'écosystème, Beneficial ecosystem processes, qui sont des processus spécifiques soutenant directement les bénéfices tirés par l'Homme (ex. production de biomasse primaire « biomasse des plantes » et secondaire "biomasse animale », épuration des eaux, régulation du climat, régulation de l'érosion, la formation des sols, etc.). Les services de l'écosystème benefits correspondent plutôt aux produits marchands ou non marchands ayant un impact direct sur le bien-être humain (alimentation, eau douce, matière primaire telle que le bois et les fibres, l'énergie " biocarburant, charbon », la santé et le bien-être psychologique). Cette classification est établie dans un objectif d'évaluation économique des écosystèmes et de la biodiversité.

La classification proposée par le Millenium Ecosystem Assessment (2005) repose sur la nature des liens entre le fonctionnement de l'écosystème et le bien-être humain et distingue quatre grandes catégories de services (de production, de régulation, culturels et de support). Elle est un outil éducatif et politique utile (Balmford $e t$ al., 2008), mais a été critiquée par Wallace (2007), Boyd et Banzhaf (2007), Fisher et al. (2009) qui soulignent qu'elle n'est pas adaptée à une évaluation économique, notamment à cause du flou des définitions des services de régulation et services support et du problème de double comptage qui se pose entre les valeurs d'usages économiques directes et indirectes de ces services. Par exemple, le service de régulation (purification de l'eau) fournit une valeur ajoutée au service support (production primaire via la purification de l'eau d'irrigation) ou au service culturel (bénéfice touristique d'une rivière propre). Ainsi, l'évaluation séparée de chaque catégorie de service mène à un double comptage de la valeur de purification de l'eau (Balmford et al., 2008). Cette limite est soulignée par Fisher et al. (2009), Balmford et al. (2008). Lavorel et Sarthou (2008) proposent trois catégories plus opérationnelles pour l'analyse économique (1) les services intrants contribuant à la fourniture de ressources et au maintien des supports physico-chimiques de la production et assurant la régulation des interactions biotiques ; (2) les services de production contribuant au revenu agricole en considérant son niveau et sa stabilité temporelle, (3) les services des produits hors revenu agricole direct, incluant le contrôle de la qualité des eaux, la séquestration du carbone ou la valeur des paysages notamment.

En agriculture, les écosystèmes de production sont gérés de manière à maximiser les services de production (Zhang et al., 2007) qui dépendent de nombreuses fonctionnalités écologiques appartenant aux services de soutien et de régulation, tels que la fertilité des sols et la pollinisation (Millenium Ecosystem Assessment, 2005). Cependant, l'agriculture est également influencée par des fonctionnalités écosystémiques néfastes «ecosystem dis-services » (Zhang et al., 2007) telles que celles assurées par les ravageurs des cultures, les adventices ou d'autres types de plantes.

Ces éléments précisent les frontières et les interactions existantes entre les fonctionnalités écologiques et les services écosystémiques, notamment en agriculture, ce qui peut complexifier l'analyse microéconomique. En effet, certaines caractéristiques des fonctionnalités écologiques telles que sa complexité et sa dynamique spatio-temporelle (hétérogène dans l'espace et évolutif dans le temps) ${ }^{2}$ sont

2. Voir Fisher et al. (2009) pour plus d'informations sur les caractéristiques des fonctionnalités écologiques. 
incompatibles avec les caractéristiques économiques d'un facteur de production tel que le travail ou le capital (mesurable en unité physique, homogène, divisible, substituable et adaptable). Bien que ces processus interviennent dans la fonction de production, les intégrer comme un facteur reste difficile puisque nous ignorons leur contribution quantitative à la production. Par exemple, la pollinisation est une fonction écologique nécessaire au service de production, mais le pollen nécessaire à la production d'un quintal de blé ainsi que sa valeur monétaire est impossible à quantifier. Au-delà, certains processus écologiques tels que la structuration des sols ou le contrôle des bio-agresseurs (habitat pour les auxiliaires) se déroulent sur un pas de temps supérieur à celui d'un cycle de production, ce qui complexifie leur intégration dans la fonction de production.

Néanmoins, la recherche des technologies et de pratiques agricoles permettant la valorisation des mécanismes écologiques et la substitution de ceux-ci, même partiellement, à certains autres inputs chimiques et phytosanitaires, est un objectif de l'AEI qu'il convient d'appréhender.

\section{Les ressources naturelles $(R N)$}

Définies par Kerry Smith et al. (1979) comme l'ensemble de «tous les éléments originaux qui composent les dotations naturelles de la terre », les RN se distinguent par leurs capacités propres de régénération et n'ont de sens que par rapport à une technologie donnée et à des conditions économiques favorables (Rotillon, 2005). On distingue (1) les ressources naturelles épuisables présentées sous la forme de stocks physiques finis (ex. énergie fossile, les minéraux) et (2) les ressources renouvelables ayant une capacité de reproduction propre, indépendante de l'intervention humaine (ex. l'eau, la fertilité des sols, la biodiversité, etc.). L'activité agricole est particulièrement concernée par l'utilisation des ressources naturelles comme le sol, l'eau, les minéraux et l'énergie fossile (carburant, engrais) et a un impact sur leur renouvellement (eau, biodiversité...).

Les économistes néoclassiques ont reconsidéré ces ressources en les intégrant comme des actifs naturels impliquant des choix d'allocation d'usage pour assurer leur disponibilité future (Faucheux et Noël, 1995). L'état des ressources naturelles et leur contribution à la production ne sont pas considérés comme un facteur de développement. Cependant, leur protection, à travers l'introduction d'une réglementation environnementale, a été intégrée, soit comme une opportunité permettant aux producteurs d'améliorer leur productivité globale en leur offrant une forte incitation à modifier leurs pratiques ou à adopter de nouvelles technologies (Porter et Van der Linde, 1995), soit comme une contrainte où les réglementations environnementales peuvent engendrer des coûts supplémentaires (Palmer et al., 1995). À la considération de la productivité s'ajoute l'analyse des effets secondaires de la production sur l'environnement et sur l'activité économique.

Ces effets non intentionnels sont formalisés par l'économie de l'environnement comme externalité et par l'économie écologique comme production jointe/ conjointe. La différence entre ces deux notions complémentaires réside dans la nature de la relation entre les agents économiques (Baumgärtner et al., 2006). En effet, l'externalité définie par Buchanan et Stubblebine (1962) implique une interdépendance des fonctions d'utilité. Selon Baumol et Aotes (1988), l'externalité est présente dès que l'activité d'un agent (A) inclut des variables réelles (non monétaires) dont les valeurs ont été choisies par d'autres sans attention particulière à leurs effets sur le bien-être de A. Une externalité négative considère le dommage environnemental comme une perte de bien-être ou d'utilité causée par un agent économique 
sur un autre en dehors d'un échange volontaire entre eux, ce qui représente une description basée sur l'effet. La production jointe considère plutôt la cause de l'effet, où souvent on observe que l'origine $\mathrm{du}$ dommage environnemental est une production non intentionnelle commune aux deux agents (Baumgärtner et al., 2006). La production jointe relève d'un processus productif qui mobilise plusieurs inputs et génère plusieurs outputs qui peuvent être des biens désirables, mais souvent, ils causent des dommages à l'environnement. Ainsi, l'utilisation de certaines ressources naturelles génère de la pollution (ex. utilisation du pétrole, émission de $\mathrm{CO}_{2}$, fertilisation minérale, lixiviation d'azote) (Baumgärtner et al., 2006, 2001). Ainsi, le rendement ne dépend pas seulement des intrants et des techniques mobilisés pour la production, mais aussi, à long terme d'autres outputs tels que l'érosion ou le nitrate lessivé. La pollution des eaux causée par les résidus d'azote est généralement analysée comme une externalité négative affectant le bien-être des consommateurs, sans faire le lien avec l'utilisation des ressources naturelles mobilisées pour la production (terre, minéraux, énergies). Il existe alors une dualité entre l'explication basée sur l'effet et l'explication à partir de la cause (Baumgärtner et al., 2006).

Dans cette logique, on trouve deux approches d'évaluation des externalités environnementales et des ressources naturelles (Louhichi et al., 2007). D'une part, l'approche classique basée sur la valorisation monétaire des dommages environnementaux qui internalise les effets externes dans la sphère marchande. Elle renvoie à l'économie de l'environnement qui s'attache essentiellement au problème des rejets engendrés par les activités économiques et considère que les problèmes environnementaux, assimilés à des gaspillages de ressources naturelles, proviennent d'une insuffisante définition des responsabilités des acteurs économiques. Cette approche suppose que les ressources échangées soient définies, protégées, exclusives et librement transférables. Ainsi, l'instauration de droits de propriété échangeables entre pollueurs et pollués (théorème de Coase), ou bien les écotaxes qui renvoient à l'analyse pigouvienne, suffiraient à résoudre le problème de l'internalisation des coûts sociaux. Or les ressources naturelles et l'environnement considérés comme biens publics et libres ne sont pas soumis aux droits de propriété et la solution décrite par Coase, par exemple, n'est applicable que dans un nombre limité de cas de pollution (Vallée, 2007). D'autre part, l'approche hétérodoxe propose une valorisation physique de l'externalité et l'intègre dans une logique d'optimisation économique sous contraintes environnementales exprimées en grandeur physique (Barde, 1991). Cette approche renvoie à l'économie écologique développée surtout dans les pays anglosaxons et scandinaves, où certains auteurs appellent à un rapprochement multidisciplinaire entre les sciences du vivant et les sciences sociales (Boulding, 1966 ; Georgescu-Roegen, 1966 ; Daly, 1968). L'évaluation physique considère donc la capacité et le rythme de reproduction des ressources renouvelables et leur risque d'épuisement. Elle est utilisée pour estimer un seuil au-dessus duquel aucun dommage ne se produit et permet de fixer des objectifs environnementaux ou des limites supérieures à ne pas dépasser pour assurer une durabilité des écosystèmes (Barde, 1991 ; Louhichi et al., 2007).

Ainsi, la démarche AEI peut reposer sur le concept de production jointe pour considérer ces ressources en termes de rareté et d'impacts environnementaux qu'engendre leur utilisation massive. Par exemple, la terre ne peut être considérée uniquement comme un support de la production, mais son niveau de fertilité doit être conservé afin de maintenir la productivité. Elle peut être altérée à long terme par l'érosion ou des dégradations agro-chimiques, d'où 
l'intérêt de considérer son utilisation en tant que facteur de production et d'intégrer sa dégradation comme une production jointe ou externalité négative. Cependant, considérer les différentes ressources naturelles comme facteur de production peut poser un problème puisque certaines ressources sont des biens sans valeur marchande et difficiles à quantifier y compris au plan technique. Pour cela, la suite de l'analyse est centrée sur les ressources naturelles marchandes qui interviennent directement dans la production.

\section{Les ressources naturelles dans les fonctions de production}

Solow (1974) et Stiglitz (1979) ont cherché à intégrer des ressources naturelles dans la fonction de production en considérant une dimension «Ressources » dans une fonction Cobb-Douglas qui compte alors trois dimensions : $\mathrm{Q}=\mathrm{K}^{\alpha 1} \mathrm{R}^{\alpha 2} \mathrm{~L}^{\alpha 3}$ avec $\mathrm{Q}$, le produit, $\mathrm{K}$ le capital, $\mathrm{R}$ le flux de ressources naturelles utilisé dans la production, $\mathrm{L}$ le travail et $\alpha_{1}+\alpha_{2}+\alpha_{3}=1$. Ils estiment qu'à population constante, une consommation soutenue de la ressource par habitant est possible si $\alpha 1>\alpha 2$, soit si l'élasticité du capital est supérieure à celle des ressources naturelles.

Cette représentation est critiquée par Georgenscu-Roegen (1975, 1979) puisqu'elle suppose une substitution infinie entre le capital et la ressource naturelle, alors que la production même du capital épuisera le stock des ressources. Ainsi, le problème de l'allocation intergénérationnelle n'est pas résolu. On note également que Stiglitz (1979) ne s'intéressait ni aux ressources renouvelables après utilisation (stocks de poisson par exemple) ni aux ressources qu'il appelait inexhaustible but nonaugmentable (comme la terre) ni aux recyclable ressources (comme l'air propre), mais plutôt aux ressources naturelles épuisables et non renouvelables comme le pétrole, le fer et le charbon. Or la production agricole nécessite à la fois des ressources renouvelables (terre, eau, air, énergie solaire) et des ressources non renouvelables comme le pétrole et les minéraux $(\mathrm{N}, \mathrm{K})$. Bien que ces minéraux soient relativement disponibles dans la nature, leurs extractions et traitements nécessitent beaucoup d'énergie.

L'intégration de la plupart de ces ressources dans la fonction de production est effectuée dans une logique d'intensification du capital par rapport à la terre. Elles sont considérées comme des consommations intermédiaires. Par ailleurs, l'AEI doit améliorer leur productivité et certaines d'entre elles comme le carburant, l'azote et le phosphate, devraient sortir de l'ensemble des charges intermédiaires et leur productivité devrait être analysée de manière spécifique.

Ainsi, une fonction de production intégrant les facteurs capital « $\mathrm{K}$ » et travail « $\mathrm{L}$ », les ressources naturelles renouvelables (RNR), terre « $\mathrm{T}$ » et eau « $\mathrm{E} »$, et non renouvelables (RNNR), carburant et engrais minéraux, prend la forme :

$$
\mathrm{Q}=\mathrm{F}(\mathrm{K}, \mathrm{W}, \mathrm{RNR}, \mathrm{RNNR}) \text { équation (1) }
$$

La contribution de l'ensemble des ressources naturelles $(\mathrm{RN})$ au processus productif peut être appréciée en moyenne $(\mathrm{Q} /$ $\mathrm{RN})$ ou à la marge $(\Delta \mathrm{Q} / \Delta \mathrm{RN})$.

Leur intégration dans une fonction de type Cobb-Douglas peut être formulée comme suit :

$$
\mathrm{Q}=\mathrm{K}^{\alpha} \mathrm{L}^{\beta} \mathrm{E}^{\gamma 1} \mathrm{~T}^{\gamma 2} \mathrm{~F}^{\gamma 3} \mathrm{G}^{\gamma 4} \text { équation (2) }
$$

Avec E la quantité d'eau d'irrigation, T la terre, $\mathrm{F}$ l'énergie fossile et $\mathrm{G}$ les fertilisants ; $\left(\alpha, \beta, \gamma_{1}, \gamma_{2}, \gamma_{3}, \gamma_{4}\right)$ sont les coefficients d'élasticité qui reflètent leur contribution dans la fonction de production.

Avec ce type de formulation, seules les variables marchandes sont considérées et certaines autres variables demeurent aujourd'hui difficiles à évaluer. On considère également que le produit $\mathrm{Q}$ ne doit pas être pris en compte en termes d'unité physique 
(Georgenscu-Roegen, 1979), mais plutôt en valeur économique (Stiglitz, 1997). Le changement de pratiques ou de technologies peut alors diminuer la quantité utilisée d'une ressource tout en maintenant la même valeur économique, ceci en augmentant l'efficience d'utilisation de cette ressource.

Un autre élément à intégrer est le facteur temporel renvoyant à l'équité intergénérationnelle. En effet, l'amélioration de la productivité des ressources naturelles renouvelables et non renouvelables doit être continue et évoluer dans un cadre de viabilité permettant une équité entre les générations. Dans cette perspective de prise en compte effective de la productivité des ressources naturelles, la démarche AEI doit être évaluée en termes d'analyse globale de l'efficience productive des exploitations agricoles et non pas uniquement en termes d'efficience de la technologie et/ ou de la pratique agricole adoptée. Ainsi, la mesure de l'efficience d'une unité de production permet de déterminer si cette dernière peut accroître sa production sans consommer plus de facteurs, ou bien réduire l'utilisation d'au moins un de ces facteurs tout en conservant le même niveau de production (Atkinson et Cornwell, 1994). Pour aller plus loin dans cette approche économique de l'AEI, nous mobilisons le concept d'efficience initié par Koopmans (1951) et Debreu (1951) et développé par Farrell (1957).

\section{L'efficacité et l'efficience pour l'analyse de la démarche AEI}

L'objectif de la démarche AEI est de conserver voire augmenter l'efficacité des exploitations agricoles, soit leur capacité à produire des quantités importantes d'aliments, mais aussi à améliorer leur efficience d'utilisation des ressources naturelles, notamment celles qui ne sont pas renouvelables.

La mesure de l'efficience d'utilisation des ressources non renouvelables telles le carburant et les engrais permet d'identifier les améliorations qui pourraient être apportées au niveau des exploitations. Il existe deux composantes de l'efficience formalisées

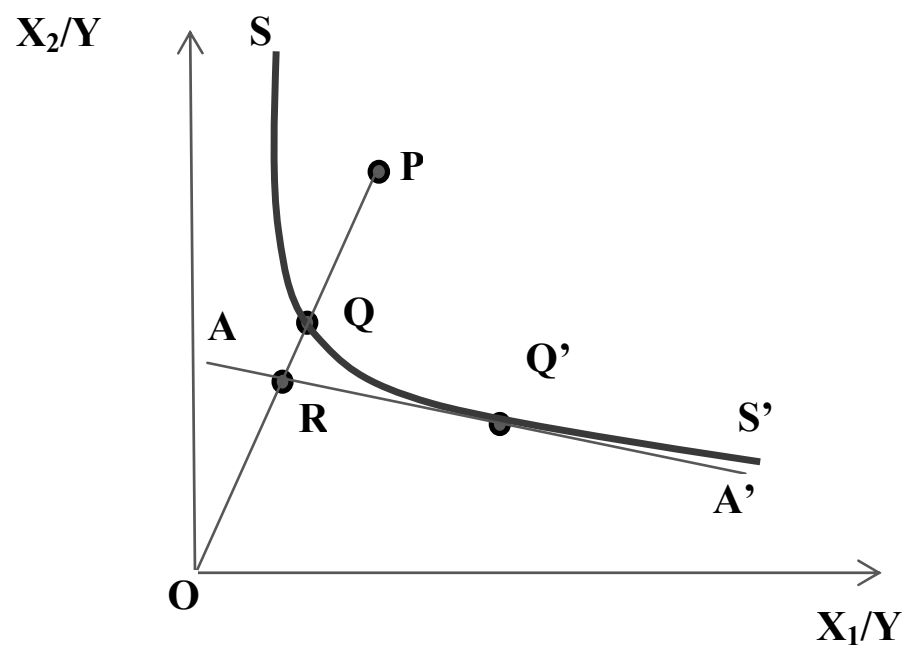

Source : d'après Farrell, 1957 


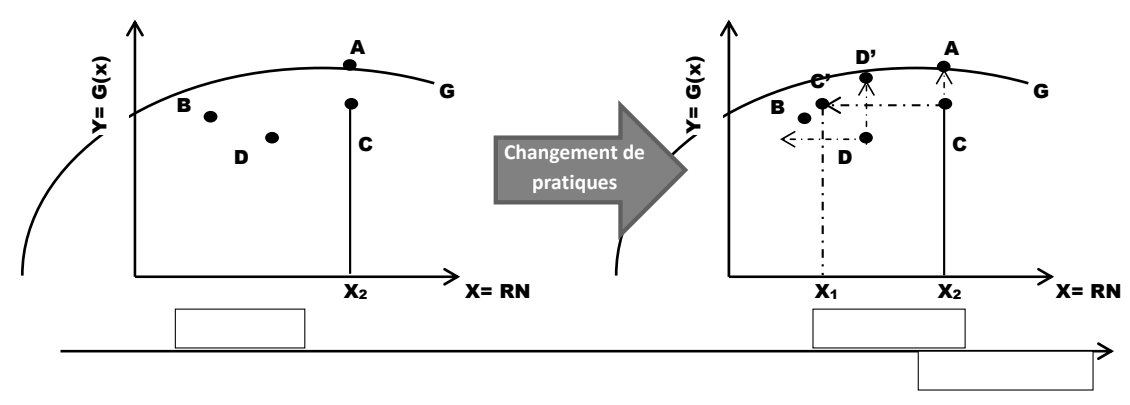

Source : les auteurs.

par Farrell (1957) : l'efficience technique (figure 2) et l'efficience allocative.

L'efficience technique (ET) correspond à une frontière de production indiquant le niveau maximum de production à réaliser avec une combinaison donnée d'intrants (SS'). Chaque combinaison d'inputs située à la frontière est techniquement efficiente ${ }^{3}$. Tout point au-dessus et à droite de celle-ci est techniquement inefficient. L'entreprise $\mathrm{P}$ est alors considérée comme techniquement inefficiente puisqu'elle peut réduire les quantités de ces deux intrants de la proportion représentée par le segment PQ. Le segment OP représente le ratio constant de l'utilisation des deux facteurs. Ainsi, la réduction potentielle pour l'entreprise $\mathrm{P}$ est déterminée de la manière suivante :

$$
\frac{P Q}{O P}=\frac{O P-O Q}{O P}=1-\frac{O Q}{O P}
$$

Le ratio $\mathrm{OQ} / \mathrm{OP}$ est défini comme étant le niveau d'efficience technique de l'entreprise P. L'efficience allocative (EA) permet d'évaluer la façon dont l'exploitant choisit la combinaison des facteurs en fonction des prix de marché et permet d'étudier le pourcentage des coûts variables réellement

3. Le terme efficacité technique est aussi utilisé pour désigner l'efficience technique. nécessaires pour produire la même quantité en modifiant les combinaisons de facteurs. Dans la figure 2, si la droite AA' représente la pente correspondant au ratio des prix des facteurs, le point Q' et non Q correspond alors au point de production allocativement le plus efficient. L'entreprise se trouvant au point $Q$ peut réduire ses coûts de production d'une proportion égale au segment RQ sans réduire son niveau d'efficience technique. En appliquant le même raisonnement de l'efficience technique, la réduction potentielle des coûts des intrants est le rapport $(\mathrm{EA}=\mathrm{OR} / \mathrm{OQ})$ qui représente l'efficience allocative de l'entreprise avec :

$$
\frac{Q R}{O Q}=\frac{O Q-O R}{O Q}=1-\frac{O R}{O Q}
$$

Afin de représenter l'intérêt de la démarche AEI, on considère une fonction de production qui tient compte d'un seul facteur de production et un seul produit ${ }^{4}$. On suppose que cette frontière est relative à l'utilisation d'une ressource naturelle à l'instant $\mathrm{T}$ et correspondant aux technologies de l'agriculture conventionnelle. Soit

4. Pour simplifier, la fonction de production présentée dans la figure 3 compte un seul facteur et un seul produit, alors que la fonction de production de l'exploitation agricole est multi-produits/multifacteurs. 
G la courbe qui définit cette relation efficiente entre le produit $\mathrm{Y}$ (rendement) et la ressource naturelle $\mathrm{X}$ (figure 3 ). Les exploitations $\mathrm{A}$ et $\mathrm{B}$, sur cette courbe, sont techniquement efficientes alors que $\mathrm{C}$ et $\mathrm{D}$ sont techniquement inefficientes, $\mathrm{C}$ utilisant la quantité X2 de ressource correspond à un processus de production inefficace puisque le niveau d'efficience pour le même niveau de la ressource permet d'être en A.

Les exploitations inefficientes ( $\mathrm{C}$ et $\mathrm{D})$ peuvent augmenter leur productivité globale par une meilleure allocation des facteurs de production ou par un changement de pratiques. Dans ce cas, les exploitations sous la frontière de production peuvent augmenter leur production pour la même quantité de facteur naturel ( $\mathrm{C}$ vers $\mathrm{A})$, ou garder la même production avec moins de ressources (C vers C'). Par exemple, si l'on considère la ressource naturelle correspondant à l'énergie fossile (carburant), le passage d'une technique de travail du sol avec labour à un travail de sol simplifié (TCS) peut réduire la quantité de gasoil utilisée de $100 \mathrm{~L} / \mathrm{h}$ a en labour à $75 \mathrm{~L} / \mathrm{ha}$ en TCS et à $50 \mathrm{~L} / \mathrm{h}$ e en semis direct. Un autre exemple concerne la valorisation des fertilisants organiques permettant de réduire l'utilisation des engrais minéraux en gardant les mêmes niveaux de production.
Dans le même registre, la modélisation des maladies du blé assistée par ordinateur permet aux agriculteurs d'adapter finement leurs fongicides, aboutissant à une économie d'énergie (moins de carburant) et une diminution totale de $20 \%$ de produits utilisés (Terrena, 2010).

Le changement de pratiques agricoles ne se fait pas sans prise de risques et la nonmaîtrise des nouvelles pratiques peut s'avérer dommageable pour les exploitations. Par exemple, les pratiques de travail de sol simplifiées sont techniquement plus compliquées et plus risquées que la pratique du labour et peuvent engendrer des pertes de rendement. Pour cette raison, la démarche AEI ne propose pas un changement brutal, mais progressif nécessitant un temps d'apprentissage pour les agriculteurs et un temps d'adaptation pour les ressources naturelles et les fonctionnalités écologiques pour rétablir une activité biologique normale. Par ailleurs, l'intérêt de développer de l'AEI dans un cadre collectif (coopérative) offre la possibilité de mutualiser ces risques pris au niveau des exploitations.

Pour les exploitations à la frontière de production (A et B), l'augmentation de la productivité des facteurs ne peut avoir lieu qu'avec un progrès technique repoussant le

Figure 4. Progrès techniques et productivité des ressources naturelles

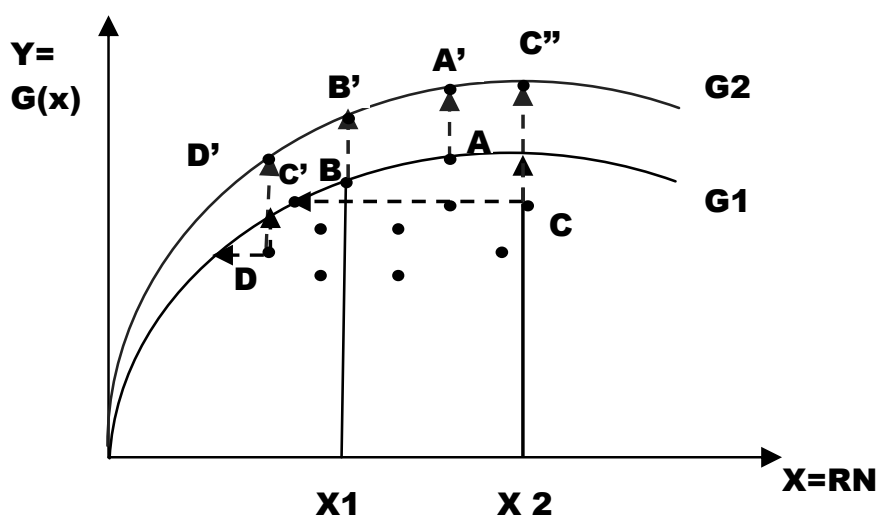

Source : les auteurs. 
stade de rendements décroissants des facteurs. Ceci se traduit par un changement de courbe de production correspondant à un état différent de la technologie (figure 4).

Il ne s'agit pas uniquement de rechercher un progrès technique permettant d'augmenter la productivité de la terre, mais de faire évoluer les processus et systèmes de production afin de réduire les effets nocifs sur l'environnement (Beise et Rennings, 2005). Ainsi, la démarche AEI devrait, dans ce cadre d'analyse, permettre aux exploitations d'obtenir avec la même quantité de ressource naturelle une quantité supérieure d'output, ce qui minimisera l'impact environnemental du processus de production. La courbe G2 correspond à une nouvelle frontière de production et les exploitations (A, B, C et D) peuvent améliorer dans le temps leur productivité globale.

Les exploitations à la frontière de production présentent une combinaison de facteurs efficiente, mais, celle-ci n'est pas forcément celle qui minimise les coûts de l'exploitation d'où l'intérêt d'étudier l'efficience allocative en intégrant une fonction de coût variable qui indique s'il est possible de se déplacer sur la même courbe, d'un point techniquement efficient vers un autre point pour lequel les coûts sont minimisés.

Considérer que l'AEI implique un accroissement de la production agricole est à prendre avec réserve pour deux raisons : la première repose sur le fait que le progrès technologique (y compris une technologie environnementale) est généralement caractérisé par un processus intégrant trois étapes : invention, innovation et diffusion (Jaffe et al., 2002 ; Del Rio, 2009). Or il n'existe pas aujourd'hui d'inventions clairement identifiées comme écologiquement intensives qui démontrent une amélioration de la production. Une deuxième raison relève de la difficulté de distinguer une technologie disponible d'une nouvelle technologie. En effet, une technologie disponible est définie comme un ensemble de techniques disponibles et le progrès technique est un changement dans cet ensemble. L'apparition d'une nouvelle technique implique un changement dans la technologie disponible. En ce sens, la technologie disponible change continuellement ce qui rend son utilité comme base de référence assez limitée. De plus, la mise en œuvre, par exemple, d'une nouvelle technologie peut influencer le calcul de l'efficience de l'exploitation et la productivité des facteurs ; cela ne dépend pas uniquement de la technologie et de sa mise en place, mais aussi des contraintes techniques et des conditions du marché (élasticité de l'offre et de la demande des produits, prix des facteurs) (Mundlak, 2001).

Ce cadre d'analyse offre une opportunité pour l'évaluation économique de l'efficience des ressources naturelles et la vérification de la faisabilité de l'intensification écologique à l'échelle de l'exploitation. Même si certaines conditions nécessaires à cette évaluation demeurent inconnues telles que l'absence de prix et de quantification physique des ressources naturelles comme la fertilité des sols ou l'azote naturel, le suivi de l'efficience globale et environnementale des exploitations peut refléter l'efficacité de la démarche AEI à travers l'analyse de l'évolution des revenus et de la productivité d'autres ressources naturelles quantifiables. Ceci semble être étroitement lié au concept d'éco-efficacité ou l'éco-efficience qui a émergé dans les années 1990 comme un concept opérationnel pour permettre une approche pratique de la durabilité (Schaltegger, 1996 in Picazo-Tadeo et al., 2011). Il a été défini par l'OCDE (1998) comme l'efficience avec laquelle les ressources écologiques sont utilisées pour répondre aux besoins de l'Homme. Ainsi, l'AEI peut être considérée comme une des voies possibles vers la durabilité et ses impacts au niveau microéconomique et macroéconomique peuvent être évalués à 
travers l'analyse de l'efficience technique, économique et environnementale.

Cet article présente les conditions d'émergence de l'AEI et ses perspectives de développement en proposant un cadre d'analyse économique adapté. Il met en évidence l'originalité du concept d'AEI qui correspond à une démarche dynamique de changement plutôt qu'un ensemble de techniques agricoles. L'AEI repose sur la valorisation des fonctionnalités écologiques et des ressources naturelles en les plaçant au centre du fonctionnement économique du secteur agricole et impose un changement dans l'analyse des systèmes de production. Cette démarche repose sur l'hypothèse forte de considérer les fonctionnalités écologiques et les ressources naturelles comme des facteurs spécifiques de production et non comme des externalités et induit un processus d'innovation et de valorisation de nouvelles pratiques issues du terrain. L'analyse économique montre qu'il est difficile actuellement de considérer les fonctionnalités écologiques comme un facteur de production à part entière étant donné les limites qu'elles présentent en termes de quantification physique et valorisation monétaire. Cependant, des analyses pluridisciplinaires reliant approches économique, sociale et environnementale telles que la modélisation bioéconomique ou l'analyse multicritères peuvent renseigner sur les impacts de certaines pratiques relevant de l'AEI à l'échelle de l'exploitation. Ces approches permettront, à travers des indicateurs économiques et environnementaux d'apprécier les effets des pratiques AEI sur les fonctionnalités écologiques de l'écosystème et de faire le lien avec l'efficacité économique.

En ce qui concerne les ressources naturelles, l'analyse théorique suggère que seules les ressources naturelles présentant les caractéristiques économiques d'un facteur de production peuvent être considérées dans la fonction de production. Pour atteindre cet objectif, il est nécessaire de développer les recherches permettant de caractériser les ressources naturelles et les fonctionnalités écologiques et l'identification de leurs relations avec les rendements, il s'agit d'identifier la relation « input-output » qui détermine la fonction de production. Il est également nécessaire d'identifier les pratiques et les technologies qui valorisent le fonctionnement des ressources naturelles en permettant l'amélioration des rendements et des performances économiques.

La mesure de l'efficacité technique des exploitations permettra d'apprécier le niveau d'efficience et de productivité des facteurs de production issus des ressources naturelles non renouvelables telles que l'énergie fossile (pétrole, minéraux) qui sont jusqu'à présent intégrées à l'ensemble des consommations intermédiaires sans établir de lien direct avec la rareté de la ressource et l'effet de sa dégradation. Elle permettra d'identifier les marges de manœuvre des agriculteurs vis-à-vis la réduction d'utilisation de ces facteurs et de cibler certaines innovations qui pourront augmenter la productivité de ces ressources. Ainsi, il deviendra possible d'analyser l'impact réel de l'AEI sur l'efficience des exploitations. Par exemple, le suivi d'un échantillon d'agriculteurs engagés dans la démarche AEI tel que les exploitations recensées dans les «sentinelles de la terre » pourrait nous renseigner sur leur efficacité productive, en comparaison avec des exploitations utilisant des techniques agricoles plus conventionnelles.

Enfin, la réussite de la démarche AEI ne dépend pas uniquement des résultats économiques obtenus, mais aussi d'autres facteurs techniques, géographiques, pédoclimatiques ou sociaux qui peuvent déterminer l'engagement des agriculteurs dans la démarche et leur manière de raisonner la relation production-environnement. 


\section{RÉFÉRENCES BIBLIOGRAPHIQUES}

Aubin J.-P. (1991). Viability Theory. Boston, Birkhäuser, 543 p.

Atkinson E. S., Cornwell C. (1994). Estimation of Output and Input Technical Efficiency Using a Flexible Functional Form and Panel Data. International Revue of Economics, n³5, p. 245-255.

Balmford A., Rodrigues A.S.L., Walpole M., Ten Brink P., Kettunen M., Braat L. De Groot R. (2008). The Economics of Biodiversity and Ecosystems: Scoping the Science. Cambridge, UK, 297 p.

Barde J.-Ph. (1991). Économie et politique de l'environnement. Paris, Éditions L'Économiste.

Baumgärtner S., Dyckhoff B., Faber M., Proops J., Schiller J. (2001). The concept of joint production and ecological economics. Ecological economics, ${ }^{\circ} 36$, p. 365-372.

Baumgärtner S., Faber M., Schiller J. (2006). Joint production and responsibility in ecological economics. On the foundations of environmental policy. Cheltenham, UK, Edward Elgar, 380 p.

Baumol W. J., Oates W.E. (1988). The theory of environemental policy. Cambridge University Press. Cambridge, $2^{\text {nd }}$ ed., 299 p.

Beise M., Rennings K. (2005). Lead markets and regulation: a framework for analyzing the international diffusion of environmental innovations. Ecological Economics, vol. 52, p. 5-17.

Boulding K.-E. (1966). The economics of the coming spaceship Earth. In H. Jarett (Ed.), Environmental Quality in Growing Economy, Baltimore, Johns Hopkins University Press, p. 3-14.

Buchanan J.-M., Stubblebine W.C. (1962). Externality. Economica, n ${ }^{\circ} 29$, p. 371-384.

Bonny S. (2011). L'agriculture écologiquement intensive : nature et défis. Cahiers Agricultures, vol. 20, $\mathrm{n}^{\circ}$ 6, p. 451-462.

Butault J.-P., Foulhouze I., Hairy D., Perraud D. (1985). Intensification et systèmes de production du lait. INSEE, p. 167-246.

Boyd J., Banzhaf S. (2007). What are ecosystem services? The need for standardized environmental accounting units. Ecological Economics, vol. 63, p. 616-626.

Cassman K. G. (1999). Ecological intensification of cereal production systems: Yield potential, soil quality, and precision agriculture. Proceedings of National Academy of Sciences, vol. 96, p. 5952-5959.

Cassman K. G. (2008). Scientific Challenges Underpinning the Food-Versus-Fuel Debate. In NABC Report 20: Reshaping American Agriculture to Meet its Biofuel and Biopolymer. Ithaca (NY), National Agricultural Biotechnology Council.

Chambers R., Pacey A., Thrupp L. A. (1989). Farmer first: farmer innovation and agricultural research. London, UK: Intermediate Technology Publications.

Daly H. E. (1968). On economics as a life science. Journal of political Economy, $\mathrm{n}^{\circ} 76$, p. 392-406.

Debreu D. G. (1951). The Coefficient of Resource Utilisation. Econometrica, $\mathrm{n}^{\circ} 19$, p. 273-292.

Del Rio P. (2009). The empirical analysis of the determinants for environmental technological change: A research agenda. Ecological Economics, vol. 86, p. 861-878.

FAO. (2009). Glossaire de l'agriculture biologique. Rome, FAO, p. 175.

Farrell M. J. (1957). The Measurement of productive Efficiency. Journal of the Royal Statistical Society. Series A (general) 120, $\mathrm{n}^{\circ} 3$, p. 253-290.

Faucheux S., Noël J.-F (1995). Économie des ressources naturelles et de l'environnement. Paris, Armand Colin, 370 p.

Fisher B., Kerry Turner R., Morling P. (2009). Defining and classifying ecosystem services for decision making. Ecological economics, vol. 68, p. 643-653.

Georgescu-Roegen N. (1966). Analytical Economics. Issues and Problems, Cambridge, MA: Harvard University Press.

Georgescu-Roegen N. (1975). Energy and economic myths. Southern Economic Journal., vol. 41, n³, p. 335-347. 
Georgescu-Roegen N. (1979). Comments on the papers by Daly and Stiglitz. p. 95-106. In V. Kerry Smith (Ed.), Scarcity and Growth reconsidered. Baltimore and London, RFF and Johns Hopkins University Press, p. 298.

Gliessman S. R. (2004). Integrating Agroecological Processes into Cropping Systems Research. In new dimensions in agroecology. D.Clements \& A. Shrestha. Binghampton, NY: Food Products Press.

Goulet F. (2012). La notion d'intensification écologique et son succès auprès d'un certain monde agricole français : une radiographie critique. Courrier de l'environnement de l'INRA, n62, p. 19-29.

Griffon M. (2007). Pour des agricultures écologiquement intensives : des territoires à haute valeur environnementale et de nouvelles politiques agricoles. Angers, Groupe ESA, Les leçons inaugurales, décembre, $73 \mathrm{p}$.

Griffon M. (2012). Les agriculteurs innovateurs : une nouvelle conception de la recherche et de l'innovation en agriculture. In Entretiens AEI (2012), Agriculture écologiquement intensive : les agriculteurs innovent, AEI association internationale pour une agriculture écologiquement intensive, Angers, $88 \mathrm{p}$.

Griffon M. (2013). Qu'est-ce que l'agriculture écologiquement intensive? Versailles, Quae, $221 \mathrm{p}$.

Jaffe A. B., Newell R. G., Stavins R. N., (2002). Environmental policy and technological change. Environment and Resource Economics, vol. 22, n¹-2, p. 41-69.

Kerry Smith V., Krutilla J. V. (1979). The economics of natural resource scarcity: an interpretive introduction. In V. Kerry Smith (Ed.), Scarcity and Growth reconsidered, Baltimore and London, RFF and Johns Hopkins University Press, 298 p.

Kiley-Worthington M. (1981). Ecological agriculture. What it is and how it works? Agriculture and Environment, $\mathrm{n}^{\circ} 6$, p. 349-381.

Koopmans T. (1951). Analysis of Production as an Efficient Combination of Activities. Edition Koopmans T.C, Activity Analysis of Production and Allocation, Cowles Commission for Research in Economics, New York, John Wiley and sons, Inc., Monograph, $\mathrm{n}^{\circ} 13$.
Lavorel S., Sarthou J.-P. (2008). Intérêts de la biodiversité pour les services rendus par les écosystèmes. In Le Roux et al., Agriculture et biodiversité. Valoriser les synergies, INRA, Expertise scientifique collective, synthèse du rapport.

Louhichi K., Boisson J.-M., Flichman G. (2007). Analyse économique de l'environnement et spécificités de la production agricole : pour une modélisation agro-économique. La Revue d'économie méridionale, vol. 55, n²20, p. 261-285.

Martinet V., Doyen L. (2007). Sustainability of an economy with an exhaustible resource: a viable control approach. Resource and Energy Economics, vol. 29, p. 17-39.

Martinet V. (2010). La «viabilité », une approche du développement durable visant à éviter les crises dans le long terme : l'exemple des pêcheries. INRA Sciences Sociales, $\mathrm{n}^{\circ} 1,4 \mathrm{p}$.

MEEDDM (ministère de l'Écologie, de l'Énergie, du Développement durable et de la Mer). (2010). Projet de caractérisation des fonctions écologiques des milieux en France. Collection Études et Documents, mai, $n^{\circ} 20$.

Millenium Ecosystem Assessment. (2005). Ecosystems and Human Well-being: Synthesis. Washington, DC, Island Press, $151 \mathrm{p}$.

Mundlak Y. (2001). Production and supply. Handbook of Agricultural Economics, vol. 1, Part A, p. 3-85.

OCDE (1998). Eco-efficiency. Paris, p. 88.

Olsson P., Folke P. (2001). Local ecological knowledge and institutional dynamics for ecosystem management: a study of Lake Racken watershed, Sweden. Ecosystems, vol. 4, p. 5-104.

Palmer K., Wallace E. O., Portney P. (1995). Tightening Environmental Standards: The benefit-Cost or the No-Cost Paradigm. Journal of Economic Perspectives, $\mathrm{n}^{\circ}$, p. 119-131.

Porter M. E., Van der Linde C. (1995). Towards a New Conception of the EnvironmentalCompetitiveness Relationship. Journal of Economic Perspectives, ${ }^{\circ}{ }^{9}$, p. 97-118.

Picazo-Tadeo A. J., Gomez-Limon J. A., ReigMartinez E. (2011). Assessing farming eco-efficiency: A Data Envelopment 
RECHERCHES

Mohamed GHALI, Karine DANIEL, François COLSON, Stéphane SORIN

Analysis approach. Journal of Environmental Management, vol. 92, p. 1154-1164.

Pretty J. (2008). Agricultural sustainability: concepts, principles and evidence. Philosophical transactions of The Royal Society B, $\mathrm{n}^{\circ} 363$, p. 447-465.

Ridier A., Ghali M., Nguyen G., Kephaliacos K. (2013). The role of risk aversion and labor constraints in the adoption of low input practices supported by the CAP green payments in cash crop farms. Review of Agricultural and Environmental Studies (à paraître).

Rotillon G. (2005). Économie des ressources naturelles. Paris, La Découverte, coll. « Repères », n406, 123 p.

Schaltegger S. (1996). Corporate Environmental Accounting. Chichester, John Wiley and Sons Ltd.

Solow R. M. (1974). The economics of resources or the resources of economics. The American Economic Review, vol. 64, n², p. 1-14.

Stiglitz J. E. (1979). A neoclassical analysis of the economics of natural resources. In V. Kerry Smith (Ed.), Scarcity and Growth reconsidered, Baltimore and London, RFF and Johns Hopkins University Press, 298 p.

Stiglitz J. E. (1997). Reply GeorgescuRoegen versus Solow/Stiglitz. Ecological Economics, $\mathrm{n}^{\circ} 22$, p. $269-270$.
Terrena (2010). Sentinelles de la terre : mettre en pratiques une agriculture écologiquement intensive. Édition $\mathrm{n}^{\circ} 11907,191$ p.

Tirel J.-C. (1983). Le débat sur le productivisme. Économie rurale, $\mathrm{n}^{\circ} 155$, p. 23-30.

Tirel J.-C. (1987). Valeur et limites des notions d'intensification dans l'analyse de l'évolution des systèmes de production. Comptes rendus de l'Académie d'Agriculture de France, $\mathrm{n}^{\circ} 73$, p. 83-95.

Uphoff N. (1998) Understanding social capital: learning from the analysis and experience of participation. In P. Dasgupta, I. Serageldin (Eds.), Social capital: a multiperspective approach Washington, DC, World Bank.

Vallée A. (2007). Les solutions économiques aux problèmes environnementaux : entre État et marché. Développement et environnement. Cahiers Français, n³37, p. 43-54.

Wallace K. J. (2007). Classification of ecosystem services: problems and solutions. Biological Conservation, vol. 139, n³-4, p. 235-246.

Zhang W., Ricketts T. H., Kremen C., Carney K., Swinton S. M. (2007). Ecosystem services and dis-services to agriculture. Ecological Economics, ${ }^{\circ} 64$, p. 253-260. 